\title{
A Multiplex Autoantibody Panel for Early Detection of Autoimmune Disease Activity
}

\author{
Yuanyuan Yang*, Karthik Krishna, Vinodh Ranganathan, Vasanth Jayaraman, \\ Tianhao Wang, Kang Bei, Hari Krishnamurthy, John J. Rajasekaran \\ Vibrant America LLC, San Carlos, CA, USA \\ Email:*shirley@vibrant-america.com
}

How to cite this paper: Yang, Y., Krishna, K., Ranganathan, V., Jayaraman, V., Wang, T., Bei, K., Krishnamurthy, H. and Rajasekaran, J.J. (2018) A Multiplex Autoantibody Panel for Early Detection of Autoimmune Disease Activity. Open Journal of Rheumatology and Autoimmune Diseases, 8, 43-52.

https://doi.org/10.4236/ojra.2018.82004

Received: February 3, 2018

Accepted: May 6, 2018

Published: May 9, 2018

Copyright $(9) 2018$ by authors and Scientific Research Publishing Inc. This work is licensed under the Creative Commons Attribution International License (CC BY 4.0).

http://creativecommons.org/licenses/by/4.0/

\begin{abstract}
Background: Detection of autoantibodies has played a consolidate role in diagnosis of systemic autoimmune disorders. A cascade autoantibody testing is usually performed by employing antinuclear antibodies (ANA) test as a first screening test and the other tests as second level determinations. Here, we present that supplementing extractable nuclear antigens (ENA) tests to the ANA test may capture more autoimmunity and provide critical medical information at an early stage. In this study, we evaluated the clinical significance of a multiplex ANA + ENA panel. Methods: A cohort of 110 subjects, identified as ANA negative but ENA positive, were followed up for two years. The detection of their ANA and anti-ENA autoantibodies was assessed with a multiplex ANA + ENA panel at Vibrant America Clinical Laboratory. Results: During two years of multi-visit follow-up, 23 out of 110 subjects (20.9\%) were found to become ANA positive within an average of $385( \pm 144)$ days. Histone $(50 / 110,45.5 \%)$ and Chromatin $(25 / 110,22.7 \%)$ antibodies were the most frequently found antibodies at their first visits. The subjects who were positive for RNP $(5 / 8,62.5 \%)$ and SSA (Ro) $(10 / 22,45.5 \%)$ have the highest ratio of conversion to positive ANA. No significant correlation was observed between the conversion frequency and the number of anti-ENA antibodies being carried. Conclusion: This study, which followed up on the subjects who had disparate ANA and ENA test results, showed that anti-ENA antibodies may exist years earlier than ANA. Combining ENA tests with ANA screening may reduce false negatives and improve sensitivity.
\end{abstract}

\section{Keywords}

Autoantibody, ANA, ENA, Autoimmunity, Connective Tissue Disorder

\section{Introduction}

The serological presence of autoantibodies is critical in early diagnosis of pa- 
tients with autoimmune disorders (e.g., systemic lupus erythematosus, Sjögren's syndrome, systemic sclerosis, rheumatoid arthritis, multiple connective tissue disorder) [1] [2]. A cascade autoantibody testing is usually performed by employing antinuclear antibodies (ANA) test as a first screening test and the other tests as second level determinations [3]. The possibility of an accurate diagnostic autoimmune disorder is closely related to the completion of ANA screening and following tests. The development of high-throughput quantitative antibody-based assays has been intensively pursued and already replaced most traditional manual qualitative assays [4]. There has been broad interest in standardizing diagnostic tests in order to predict the development of diseases, reduce the cost of repeated confirmatory tests, and avoid unnecessary continued investigations [3] [5].

ANA is a heterogenous group of autoantibodies that can be found in the serum of patients with systemic or organ specific autoimmune diseases and a variety of infections. The gold standard of ANA testing accepted by American college of Rheumatology (ACR) is immunofluorescence assay (IFA) on human epithelial type 2 (HEp-2) cells [6]. IFA identifies a variety of antigenic residues, which react to not only autosomal antibodies but also nuclear or nucleoplasmic targets in sera. While IFA detects many nuclear and cytoplasm antigens, its sensitivity and specificity in diagnosis of autoimmunity have been inherently limited by a few factors [3] [7]. First, ANA by IFA can be subjective due to its heavy dependence on humans' operation (a large number of serial dilutions) and interpretation (visual determination of staining patterns) [8]. Another significant limitation is the existence of false positives due to ANA's presence in other diseases, infections, tumors, and in $25 \%$ healthy individuals [9]. Interpretation of ANA pattern also presents a unique challenge to provide accurate and inclusive results for physicians to make decisive diagnosis.

Extractable nuclear antigens (ENA) are analyzed in order to determine the specificity of autoantibodies that have produced a positive ANA with a homogeneous or speckled pattern. Detection of anti-ENA antibodies, as a second-tier test, is usually followed by a positive ANA test to identify and distinguish between different autoimmune diseases, especially connective tissue disorders [10]. The presence of each anti-ENA antibody may either confirm or exclude a diagnosis. However, in most cases, ENA tests will not be ordered when a person has a negative ANA by IFA. From a cost-benefit point of view, ANA test possesses most of the characteristics to be employed as a first screening test. However, a multiplex test panel can have great potential serving as one of the resolutions to reduce false negatives and diagnostic time [3] [8] [11]. The progressively-developed biomedical industry may contribute technological solutions to improve the process, not only in preparing substrates and slides, but also in providing a comprehensive yet affordable test panel.

Though important, the clinical significance of a multiplex autoantibody panel in diagnosis of autoimmune disorders has been barely investigated [12]. In this study, we defined the cohort of patients to a group of 110 subjects who had dis- 
parate ANA and ENA result and followed up for two years. These subjects were negative in ANA test but showed positive to at least one anti-ENA antibodies at their first visit. During two years, 23 (20.9\%) of them converted to ANA positive in an average ( \pm standard deviation) of $385( \pm 144)$ days while they all remained to be ENA positive. This comparative study showed that a combined test of ANA and ENA was more sensitive than detecting ANA alone but also recognized autoimmune disease activity at an earlier stage.

\section{Materials and Methods}

\subsection{Patient Selection and Study Design}

From the subjects who have been addressed to the Vibrant America Clinical Laboratory for a routine testing of ANA and anti-ENA antibodies between January 2015 and July 2017, we selected all 110 unique subjects who meet the following criteria and analyzed their de-identified clinical data. Three main inclusion criteria were: 1) ANA and anti-ENA antibodies tested for at least three times during the two and a half years; 2) negative ANA test at the first visit; 3 ) consistently positive for at least one anti-ENA antibodies. The exclusion criteria were: 1) incomplete test result; 2) tested for less than three times during the two and a half years at the Vibrant America Clinical Laboratory. Clinical data were obtained by retrospective review of the de-identified test results; the subjects involved in the study are non-traceable. Mean age ( \pm standard deviation) of the subjects was 58 \pm 17 years. The female to male ratio was 1:1 ( $52 \%$ female, $48 \%$ male).

\subsection{ANA Detection}

ANA detection was performed by the Vibrant ${ }^{\mathrm{TM}}$ ANA HEp-2 (Vibrant America, LLC, San Carlos, CA, USA), which is a solid phase bio-chip immunofluorescence assay designed to detect antinuclear antibodies. Samples were incubated with antigen substrate and unreacted antibodies were washed off by a wash solution. The substrate was incubated with specific fluorescent dye labeled conjugate and then unbound reagent was washed off. Microarray chip reading was performed on a fluorescent microscope scanner, which then transmitted data to the proprietary software program for analysis. When viewing through a fluorescence microscope, autoantibody positive samples exhibited a bright fluorescence corresponding to areas of the cell or nuclei where autoantibody was bound. The Hamilton Microlab STAR robotic pipetting station used VENUS Two software programming for sample and reagent pipetting and management of plate handling, minimizing assay contamination.

The interpretation of the results depended on the pattern observed, the titer of the autoantibody, and the age of the patient [12]. A sample was considered ANA negative (ANA-) if specific staining was equal to or less than a negative control (buffer containing preservative and human serum with no IgG antinuclear antibodies). Samples might exhibit various degrees of background staining due to heterophile antibodies or low-level autoantibodies to cytoplasmic constituents 
such as contractile proteins. A sample was considered ANA positive (ANA+) if any specific staining (homogeneous, centromere, speckled, nucleolar, peripheral) was observed to be greater than the negative control. The elderly, especially women, are prone to develop low-titered autoantibodies in the absence of clinical autoimmune disease. A 1:40 dilution was suggested as a good dilution to screen for ANA [6]. Low-titer positive results might occur in apparently healthy persons; therefore, the ANA results were always interpreted considering the patients' total clinical presentation.

\subsection{ENA Antibody Detection}

Ten anti-ENA antibodies including SSA(Ro), SSB(La), RNP/Sm, Jo-1, Sm, Scl-70, Chromatin, Centromere, Histone, and RNA polymerase III were tested. SSA(Ro), SSB(La), RNP/Sm, and Jo-1 were detected using the Vibrant ${ }^{\mathrm{TM}}$ ENA-4 (Vibrant America, LLC, San Carlos, CA, USA), which is a solid phase bio-chip immunofluorescence assay that reports qualitative and semi-quantitative results for IgG to SSA(Ro), SSB(La), RNP/Sm, Jo-1. Patient results were interpreted by comparison with calibrators, controls and cut-off values.

The assessment and interpretation of the results was following the international guideline announced by the European autoimmunity standardization initiative and the International Union of Immunologic Societies/World Health Organization/Arthritis Foundation/Centers for Disease Control and Prevention autoantibody standardising committee [12]. A sample was considered ENA negative (ENA-) if the concentration of the antibody to ENA was equal to or less than the cut-off value. A sample was considered ENA positive (ENA+) when the it has at least one autoantibody to ENA at borderline of or more than an index value of 0.95. The results for Sm, Scl-70, Chromatin, Centromere, Histone, and RNA polymerase III were obtained with a commercially available ELISA kit. A positive result was decided when units value was more than 20 , which is a weak positive result suggested by the ELISA provider company.

\subsection{Statistical Analysis}

Clinical data from the de-identified subjects were included in a randomized database that was processed and analyzed using Java for Windows version 1.8.45. Data were expressed as mean \pm standard deviation (SD) when the distribution was Gaussian. Two-sample t-test is used to examine the significance of difference in two groups. $\mathrm{P}<0.05$ was considered significant.

\section{Results}

\subsection{Early Detection of Anti-ENA Antibodies Predicted ANA Conversion}

Between January 2015 and July 2017, a cohort of 110 subjects suspected with autoimmune disorders were selected to be followed up on their ANA and ENA tests using a combined autoantibody panel. This cohort was chosen based on 
their initial index test showing negative for ANA but positive for ENA (ANA-/ENA+). A total of 363 sera samples from these 110 subjects were tested in two and a half years with an average of 2.3 follow-up tests per patient (range: 2 to 4 repeats). The average interval between tests ( \pm standard deviation) was 181 $( \pm 87)$ days. As shown in Figure 1, out of the 253 follow-up tests in the 110 subjects, $34(13.4 \%)$ tests and $23(20.9 \%)$ subjects converted to ANA positive after an average ( \pm standard deviation) of $385( \pm 144)$ days while they remained to be ENA positive (ANA+/ENA+). $4(3.6 \%)$ of the 23 subjects converted to ANA positive within the first half year, $7(6.4 \%)$ converted within the second half year, and $12(10.9 \%)$ converted in the second year. The remaining 87 subjects continued to be ANA negative and ENA positive (ANA-/ENA+); however, it is expected that an increasing portion of the subjects would convert to ANA positive after a longer period of time [13].

\subsection{Prevalence of Anti-ENA Antibodies Detected in ANA Negative Subjects}

As shown in Table 1, among the 110 subjects who were ANA negative by IFA, Histone (45.5\%) and Chromatin (22.7\%) antibodies were the most frequently found antibodies at their first visit. Antibodies against Jo-1 (5.5\%) and Scl-70 (3.6\%) were the least prevalent. The other six anti-ENA antibodies were all detected in this cohort. After two years of follow-up, the subjects who were positive for RNP (62.5\%) and SSA (Ro) (45.5\%) have the highest ratio of conversion to ANA positivity. The subjects with positive RNA polymerase III (9.1\%) and Scl-70 (0\%) were among the lowest frequencies of conversion. Even though the subjects with Histone antibodies were of the highest prevalence at their first visit, only 5 of them (10\%) converted to ANA positive in two and a half years.

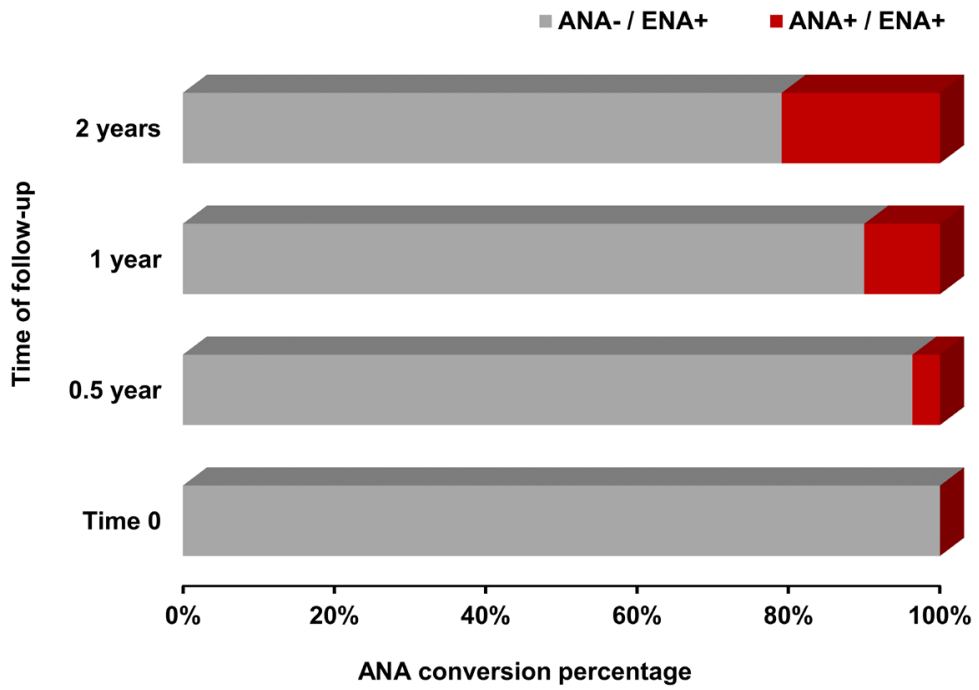

Figure 1. Out of 110 subjects who were ANA negative but had anti-ENA antibodies (ANA-/ENA+), 23 subjects (20.9\%) sero-converted to ANA positive and continued to have anti-ENA antibodies $(\mathrm{ANA}+/ \mathrm{ENA}+)$ in two years. 
Table 1. ENA autoantibodies prevalence and frequency of ANA conversion.

\begin{tabular}{ccccc}
\hline ENA & \multicolumn{2}{c}{ Prevalence at the first visit } & \multicolumn{2}{c}{ Frequency of ANA conversion } \\
\hline Histone & $50 / 110$ & $45.5 \%$ & $5 / 50$ & $10.0 \%$ \\
Chromatin & $25 / 110$ & $22.7 \%$ & $3 / 25$ & $12.0 \%$ \\
SSA (Ro) & $22 / 110$ & $20.0 \%$ & $10 / 22$ & $45.5 \%$ \\
Sm & $15 / 110$ & $13.6 \%$ & $3 / 15$ & $20.0 \%$ \\
RNA POL III & $11 / 110$ & $10.0 \%$ & $1 / 11$ & $9.1 \%$ \\
RNP & $8 / 110$ & $7.3 \%$ & $5 / 8$ & $62.5 \%$ \\
SSB (La) & $8 / 110$ & $7.3 \%$ & $2 / 8$ & $25.0 \%$ \\
Centromere & $8 / 110$ & $7.3 \%$ & $1 / 8$ & $12.5 \%$ \\
Jo-1 & $6 / 110$ & $5.5 \%$ & $2 / 6$ & $33.3 \%$ \\
Scl-70 & $4 / 110$ & $3.6 \%$ & $0 / 4$ & $0.0 \%$ \\
\hline
\end{tabular}

\subsection{No Correlation between ANA Conversion and Carrying Multiple ENA Antibodies}

The patients with more than one anti-ENA antibodies are normally highly suspected to have overlapped autoimmune disorders [14] [15]. Figure 2 however shows that the possibility of conversion from ANA negative to ANA positive was not correlated with the total number of anti-ENA antibodies being carried. Among the 110 subjects, 74 subjects had only one anti-ENA antibody and 15 (20.3\%) of them converted to ANA positive. 25 subjects had two types of anti-ENA antibodies and 7 of them (28.0\%) became ANA positive. However, only 1 out of 11 subjects $(9.1 \%)$ that who had three types of anti-ENA antibodies sero-converted to positive ANA. The p-value among these three groups were 0.4 (group 1-group 2), 0.2 (group 2-group 3), and 0.3 (group 1-group 3). Therefore, no significant difference was observed between the conversion frequency and the number of anti-ENA antibodies being carried.

\section{Discussion}

Genetic background and environmental factors (e.g., smoking, vitamin D, toxins, diet, infection, antibiotics, dysbiosis) are believed to triggering autoimmunity and inducing pro-inflammatory syndromes [16] [17]. Early detection of autoantibodies has always played an important role in predicting and diagnosing autoimmune disorders, especially for the patients suspected with overlapped syndromes and complex conditions. Improved method such as IFA for ANA screening has already greatly reduced the number of false negatives [5]. From our observation, a significant number of subjects still fall into this category, which should not be overlooked. Simultaneous detection of multiple autoantibodies has become technically and costly feasible owing to the advancement in semiconductor methods and the generation of high-throughput biotechnology. In this study, a multiplex ANA + ENA panel enabled a long-term follow-up for 110 subjects who had discrepant ANA and ENA results. While comparable 


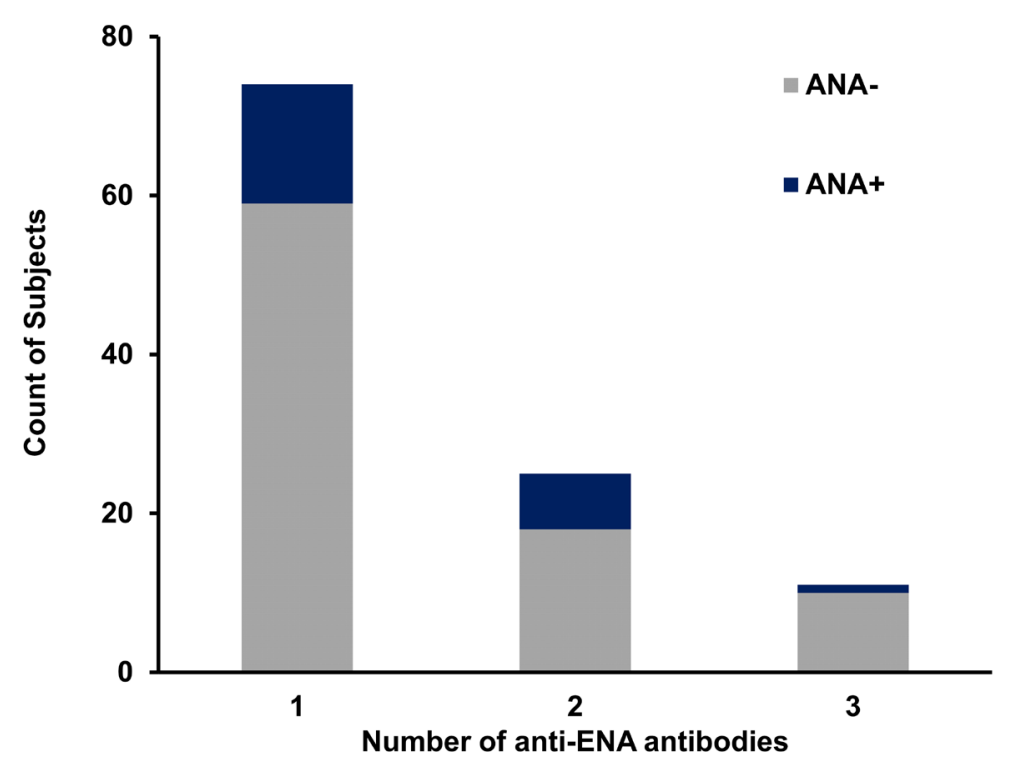

Figure 2. No correlation was observed between the conversion frequencies and the number of anti-ENA antibodies being carried.

studies of multiplex autoantibody panels have been conducted by different research groups [18] [19] [20] [21], we extended the conclusions and validated the assumptions with our microarray platform.

The most relevant consequence of performing a multiplex ANA + ENA panel is its ability to predict autoimmune disease by detecting early autoantibodies. This study confirms that anti-ENA bodies could be identified prior to the presence of ANA in some subjects. Among 110 subjects with negative ANA and positive ENA serology, 21\% developed to positive ANA during the 2.5-year follow-up. This conversion rate is in agreement with the numbers reported in another study where it was $\sim 25 \%$ for two years and $\sim 76 \%$ for three years [13]. The investigation of the same cohort is still undergoing at Vibrant America Clinical Laboratory and it would be mandatory to perform a long-term follow-up for confirmation. We have also noticed a consistent conversion frequency of $10 \%$ per year, which may provide information in predicting the percentage of false negatives in further studies. Even though a decisive confirmation of disease status were not provided for these subjects, the presence of specific autoantibodies is still among the most robust evidence to predict clinical onset of autoimmune diseases.

Our analysis showed no significant correlation between the type of anti-ENA antibody and the possibility of sero-conversion. The most prevalent anti-ENA antibody found in the subjects is anti-Histone antibodies (45.5\%) but only $10.0 \%$ of them converted to ANA positive in two and a half years. The anti-ENA antibodies associated with the highest conversion frequency are the ones to against RNP (62.5\%) but the prevalence of their carrier was only 7.3\%. Even though anti-ENA antibodies may exist in healthy people thus have limited clinical value in predicting autoimmune diseases, their presence can always be interpreted with 
symptoms to provide critical information in diagnosis. Our finding demonstrates that detecting ten types of anti-ENA antibodies rather than several specific types is advantageous. An ENA panel of ten autoantibodies provided more inclusive results and higher possibility to capture false negatives that could be missed by ANA screening.

The results from our study indicated that the frequency of ANA sero-conversion is hardly related to the number of types of anti-ENA antibodies being carried. The subjects with two types of anti-ENA antibodies had relatively higher conversion frequency (28\%) compared with the ones had either one or three types of anti-ENA antibodies. There has been relevant study showing that the average number of types of autoantibody increased with the time of diagnosis of systemic lupus erythematosus [22]. We hypothesize the difference might be due to the presence of multiple autoimmunity syndromes besides systemic lupus erythematosus in this cohort of patients and the development pattern for those syndromes can be less relevant with time. However, we believe it would be worthwhile extending this study to a larger population of subjects suspected with systemic autoimmune disorders.

\section{Conclusion}

In summary, this study showed that a combined test of ANA and ENA has great potential to reduce the number of false negatives and improve early detection of asymptomatic subjects suspected with systemic autoimmune disorders. A microarray-based autoantibody panel can detect and confirm multiple autoantibodies in one step while adhering to the standard IFA methodology and conventional ENA interpretation. Early detection at such platform will provide insights into specificity of autoantibodies present, indications of disease likelihood, and confirmation of clinical suspicion.

\section{Acknowledgements}

We acknowledge Vibrant America LLC for supporting this research.

\section{Declaration of Conflict of Interest}

Yang is employee of Vibrant America LLC. Krishna, Ranganathan, Jayaraman, Wang, Bei, Krishnamurthy, and Rajasekaran are employees of Vibrant Sciences LLC.

\section{References}

[1] Naparstek, Y. and Plotz, P.H. (1993) The Role of Autoantibodies in Autoimmune Disease. Annual Review of Immunology, 11, 79-104. https://doi.org/10.1146/annurev.iy.11.040193.000455

[2] Lleo, A., Invernizzi, P., Gao, B., et al. (2010) Definition of Human AutoimmunityAutoantibodies versus Autoimmune Disease. Autoimmunity Reviews, 9, A259-A266. https://doi.org/10.1016/j.autrev.2009.12.002 
[3] Meroni, P.L. and Schur, P.H. (2010) ANA Screening: An Old Test with New Recommendations. Annals of the Rheumatic Diseases, 69, 1420-1422. https://doi.org/10.1136/ard.2009.127100

[4] Bizzaro, N., Antico, A., Platzgummer, S., et al. (2014) Automated Antinuclear Immunofluorescence Antibody Screening: A Comparative Study of Six Computer-Aided Diagnostic Systems. Autoimmunity Reviews, 13, 292-298. https://doi.org/10.1016/j.autrev.2013.10.015

[5] Damoiseaux, J.G. and Tervaert, J.W. (2006) From ANA to ENA: How to Proceed? Autoimmunity Reviews, 5, 10-17. https://doi.org/10.1016/j.autrev.2005.05.007

[6] Position Statement: Methodology of Testing for Antinuclear Antibodies. American College of Rheumatology.

https://www.rheumatology.org/Portals/0/Files/Methodology\%20of\%20Testing\%20 Antinuclear\%20Antibodies\%20Position\%20Statement.pdf

[7] Meroni, P.L., Bizzaro, N., Cavazzana, I., et al. (2014) Automated Tests of ANA Immunofluorescence as Throughput Autoantibody Detection Technology: Strengths and Limitations. BMC Medicine, 12, 38. https://doi.org/10.1186/1741-7015-12-38

[8] Bizzaro, N. and Wiik, A. (2004) Appropriateness in Anti-Nuclear Antibody Testing: From Clinical Request to Strategic Laboratory Practice. Clinical and Experimental Rheumatology, 22, 349-355.

[9] Satoh, M., Chan, E.K., Ho, L.A., et al. (2012) Prevalence and Sociodemographic Correlates of Antinuclear Antibodies in the United States. Arthritis \& Rheumatology, 64, 2319-2327. https://doi.org/10.1002/art.34380

[10] Van Blerk, M., Bossuyt, X., Humbel, R., et al. (2014) Belgian Recommendations on ANA, Anti-dsDNA and Anti-ENA Antibody Testing. Acta Clinica Belgica, 69, 83-86. https://doi.org/10.1179/2295333714Y.0000000010

[11] Wiik, A.S. and Bizzaro, N. (2012) Missing Links in High Quality Diagnostics of Inflammatory Systemic Rheumatic Diseases. Autoimmunity Highlights, 3, 35-49. https://doi.org/10.1007/s13317-012-0029-0

[12] Agmon-Levin, N., Damoiseaux, J., Kallenberg, C., et al. (2014) International Recommendations for the Assessment of Autoantibodies to Cellular Antigens Referred to as Anti-Nuclear Antibodies. Annals of the Rheumatic Diseases, 73, 17-23. https://doi.org/10.1136/annrheumdis-2013-203863

[13] Pérez, D., Gilburd, B. and Cabrera-Marante, Ó. (2017) Predictive Autoimmunity Using Autoantibodies: Screening for Anti-Nuclear Antibodies. Clinical Chemistry and Laboratory Medicine. https://doi.org/10.1515/cclm-2017-0241

[14] Sharp, G.C., Irvin, W.S., Tan, E.M., et al. (1972) Mixed Connective Tissue Disease-An Apparently Distinct Rheumatic Disease Syndrome Associated with a Specific Antibody to an Extractable Nuclear Antigen (ENA). American Journal of Medicine, 52, 148-159. https://doi.org/10.1016/0002-9343(72)90064-2

[15] Phan, T.G., Wong, R.C.W. and Adelstein, S. (2002) Autoantibodies to Extractable Nuclear Antigens: Making Detection and Interpretation More Meaningful. Clinical and Vaccine Immunology, 9, 1-7. https://doi.org/10.1128/CDLI.9.1.1-7.2002

[16] Gianchecchi, E. and Fierabracci, A. (2015) Gene/Environment Interactions in the Pathogenesis of Autoimmunity: New Insights on the Role of Toll-Like Receptors. Autoimmunity Reviews, 14, 971-983. https://doi.org/10.1016/j.autrev.2015.07.006

[17] Muniz Caldas, C.A. and Freire de Carvalho, J. (2012) The Role of Environmental Factors in the Pathogenesis of Non-Organ-Specific Autoimmune Diseases. Best Practice \& Research Clinical Rheumatology, 26, 5-11. https://doi.org/10.1016/j.berh.2012.01.010 
[18] Op De Beeck, K., Vermeersch, P., Verschueren, P., et al. (2012) Antinuclear Antibody Detection by Automated Multiplex Immunoassay in Untreated Patients at the Time of Diagnosis. Autoimmunity Reviews, 12, 137-143. https://doi.org/10.1016/j.autrev.2012.02.013

[19] Desplat-Jego, S., Bardin, N., Larida, B. and Sanmarco, M. (2007) Evaluation of the BioPlex 2200 ANA Screen for the Detection of Antinuclear Antibodies and Comparison with Conventional Methods. Annals of the New York Academy of Sciences, 1109, 245-255. https://doi.org/10.1196/annals.1398.030

[20] Shanmugam, V.K., Swistowski, D.R., Saddic, N., et al. (2011) Comparison of Indirect Immunofluorescence and Multiplex Antinuclear Antibody Screening in Systemic Sclerosis. Clinical Rheumatology, 30, 1363-1368.

https://doi.org/10.1007/s10067-011-1766-6

[21] Hanly, J.G., Su, L., Farewell, V. and Fritzler, M.J. (2010) Comparison between Multiplex Assays for Autoantibody Detection in Systemic Lupus Erythematosus. Journal of Immunological Methods, 358, 75-80. https://doi.org/10.1016/j.jim.2010.04.005

[22] Arbuckle, M.R., McClain, M.T., Rubertone, M.V., et al. (2003) Development of Autoantibodies before the Clinical Onset of Systemic Lupus Erythematosus. The New England Journal of Medicine, 349, 1526-1533.

https://doi.org/10.1056/NEJMoa021933 